J. Lake Sci. (湖泊科学), 2011, 23(2):217-222

http: //www. jlakes. org. E-mail : jlakes@niglas.ac.cn

(C) 2011 by Journal of Lake Sciences

\title{
Lee 生物光学模型在不同水体组分特性下的适用性
}

\author{
王胜强, 陈 晋**, 杨 伟, 梁涵玮, 朱晶晶 \\ ( 北京师范大学地表过程与资源生态国家重点实验室, 北京 100875)
}

\begin{abstract}
摘 要: 辐射传输模型和生物光学模型均可用于模拟水体遥感反射率. 前者模拟精度高, 但计算复杂, 不利于水质参数的 反演; 后者简便易反演, 但在浑浊水体中的模拟精度还有待进一步检验. 本文通过设计大量不同组分浓度组成的水体, 以 辐射传输模型 (即 Hydrolight 模型) 模拟结果为真值, 对生物光学模型 (即 Lee 模型) 模拟二类水体遥感反射率的精度进行 了检验. 结果表明: (1) 除藻类悬浮物主导型水体外, Lee 模型在所有波段的总体模拟误差在 10\% 以内; (2) 叶绿素 a 浓 度反演算法常用波段光谱模拟误差较大; (3) 刚好在水面以上与刚好在水面以下的遥感反射率显示出相似的模拟精度.

关键词: 遥感反射率; Hydrolight 模型; Lee 生物光学模型
\end{abstract}

\section{Accuracy assessment of bio-optical model in turbid case II waters}

\author{
WANG Shengqiang, CHEN Jin, YANG Wei, LIANG Hanwei \& ZHU Jingjing \\ (State Key Laboratory of Earth Surface Processes and Resource Ecology, Beijing Normal University, Beijing 100875, P. R. \\ China)
}

\begin{abstract}
Both radiative transfer model and bio-optical model can be used to simulate the remote sensing reflectance for waters. Generally, the radiative transfer model is of high accuracy, but it is too sophisticated to retrieve water quality parameters (WQPs). The bio-optical model is simple and easy to be implemented in retrieving WQPs, but its accuracy in simulating turbid case II waters is still unknown. In this study, the radiative transfer model (i. e. Hydrolight model) and bio-optical model (i. e. Lee model) were both used to generated a large number of spectra data with different combinations of WQPs. Using the spectra yielded by Hydrolight model as true value, we assessed the simulation accuracy of the Lee model in turbid case II waters. Results show that (1) except for the non-phytoplankton suspended substance dominated waters, results of Lee model can be acceptable with overall relative error less than $10 \%$; (2) larger errors in Lee model occurred in the bands for Chl. a estimation than other bands; (3) simulation accuracy of remote sensing reflectance just below water surface is consistent with that just above surface.
\end{abstract}

Keywords: Remote sensing reflectance; Hydrolight model; Lee deep-water bio-optical model

水体反射率光谱在水质遥感监测中具有重要意义. 多种叶绿素估测方法, 如苂光峰位法 ${ }^{[1]}$ 、苂光峰高 法 ${ }^{[2]}$ 、面积法 ${ }^{[3]}$ 、波段比值法 ${ }^{[4]}$ 都是基于水体反射率光谱特征提出的. 一般而言, 水体反射率光谱可以通过 实地测量获得, 然而实地测量易受天气、水体表面 (波浪) 等状况的影响, 费时费力. 同时受采样点数量和组 分浓度限制, 常常不能获得建模所需的特定水体组分浓度下的反射率光谱. 因此, 通过模拟方法获得不同水 体组分浓度水体反射率光谱在水质遥感监测中具有广泛应用价值. 模拟水体反射率光谱可以基于水体辐射 传输理论而得到, 其典型代表为 Hydrolight 模型. 它具有很好的物理依据, 模拟精度高, 但计算速度慢,很难 满足产生大量模拟光谱数据的要求, 且对水体组分生物光学特性与对应的反射率光谱之间的关系没有简单 明确的数学表达式描述,其参数不具有直接可反演性.

于是, 人们提出了各种形式简单且可以用于水质参数反演的生物光学模型. Gordon ${ }^{[5]}$ 等基于二向流近 似提出的水体反射率和固有光学特性之间的简单数学关系成为了水体生物光学模型的原型. 1998 年 Lee ${ }^{[6]}$

* 国家自然科学基金项目 (40871162)资助. 2010-03-08 收稿;2010-05-25 收修改稿. 王胜强,男,1986 年生,硕士 研究生;E-mail: wsqiang815@gmail.com.

** 通讯作者;E-mail: chenjin@ ires. cn. 
等提出了适用于二类水体的生物光学模型 (以下简称为 Lee 模型), 并基于 Hydrolight 软件实现了模型参数 化, 且在水质遥感中得到广泛的应用 ${ }^{[7-10]}$. 然而, Lee 模型在基于 Hydrolight 软件参数化过程中, 水体组分为 纯水、浮游植物、可溶性有机物, 且仅选取了有限几种低组分浓度的水体, 叶绿素浓度最高为 $5.0 \mathrm{mg} / \mathrm{m}^{3}$. 研 究显示, 内陆湖泊水体的组分浓度往往远大于这个浓度值, 如马荣华等 2003 年在太湖的调查结果显示其叶 绿素 a 浓度范围为 $7.77-71.58 \mathrm{mg} / \mathrm{m}^{3[11]}$. 对于超出原模型参数化浓度范围的浑浊水体, Lee 模型的模拟精 度还有待进一步的验证.

基于以上实际应用的需求和现有工作的不足, 本文利用 Lee 模型模拟了大量含有不同组分浓度的水体 遥感反射率, 并以 Hydrolight 模型的结果为真值分析了 Lee 模型的模拟精度, 并给出其适用性条件.

\section{Lee 生物光学模型简介}

表 1 Hydrolight 模型输人参数 (Lee 模型参数化)

Tab. 1 Input parameters used in the Hydrolight simulations (Lee model parameterization)

\begin{tabular}{cc}
\hline 输人参数 & 输人量 \\
\hline 太阳天顶角 & $0^{\circ}, 30^{\circ}, 60^{\circ}$ \\
颗粒相位函数 & Petzold average particle \\
叶绿素 a 浓度 $\left(\mathrm{mg} / \mathrm{m}^{3}\right)$ & $0.4,1.0,2.0,5.0$ \\
可溶性有机物在 $440 \mathrm{~nm}$ & $0.05,0.1,0.3$ \\
处吸收系数 $a_{\mathrm{g}}(440)\left(\mathrm{m}^{-1}\right)$ & \\
$B($ 浑浊度参数 $)$ & $0.3,1.0,5.0$ \\
$\lambda(\mathrm{nm})$ & $400-700$, 每隔 20 \\
\hline
\end{tabular}

Lee 等 $^{[6]}$ 经过理论推导, 认为对于无限深水体, 星下点刚好处于水面以下的遥感反射率可以表示为:

$$
r_{\mathrm{rs}}=\left(g_{0}+g_{1} u^{g_{2}}\right) u
$$

其中, $r_{\mathrm{rs}}$ 为星下点刚好处于水面以下的遥感反射比, $u=b_{\mathrm{b}} /\left(a+b_{\mathrm{b}}\right), b_{\mathrm{b}}$ 为水体总后向散射系数, $a$ 为水体 总吸收系数, $g_{0} 、 g_{1}$ 为常数. 利用 Hydrolight 模型基于 表 1 所示参数将模型参数化, 其中 $B$ 为颗粒散射系数 表达式中的参数, 散射系数为: $b_{\mathrm{p}}(\lambda)=B[\mathrm{Chl} . \mathrm{a}]^{0.62}$ $(550 / \lambda), B$ 的大小可表示水体浑浊程度, $B$ 越大表示 水体越浑浊, 反之亦然. 本文将 $B$ 称为水体浑浊度参 数. 模型参数化后可以表示为:

$$
r_{\mathrm{rs}}=\left(0.070+0.115 u^{0.752}\right) u
$$

类似, 提出了刚好处于水面以上遥感反射比 $\left(R_{\mathrm{rs}}\right)$ :

$$
R_{\mathrm{rs}}=0.518 r_{\mathrm{rs}} /\left(1-1.562 r_{\mathrm{rs}}\right)
$$

后来 Lee 等 ${ }^{[7]}$ 对其模型参数进行了修改,修改后可表示为:

$$
r_{\mathrm{rs}}=(0.084+0.170 u) u, R_{\mathrm{rs}}=0.5 r_{\mathrm{rs}} /\left(1-1.5 r_{\mathrm{rs}}\right)
$$

\section{2 实验设计}

由 Lee 模型参数化时输人变量可以看出, 水体组分仅为浮游植物、可溶性有机物及纯水, 其叶绿素 a 浓 度 (Chl. a)、可溶性有机物 (CDOM) 浓度均较低, 且每个变量只有 3-4 个输人量. 另外, 其浑浊度参数 $B$ 的定 义较为模糊. 为了研究 Lee 模型在水体组分浓度更大变化范围内的适用性及模拟误差与各个组分的关系, 本 实验设计 4 种不同组分的水体,每种水体对应不同范围的组分浓度. 为与目前对二类水体四组分 (即纯水、 浮游植物、可溶性有机物、非藻类悬浮物) 的定义保持一致, 用非藻类悬浮物 (NPSS) 浓度代替浑浊度 $B, 4$ 种 类型水体对应组分浓度见表 2 , 其余参数设置与表 1 类似, 太阳高度角为 $30^{\circ}$; 浮游植物其相位函数为 Petzold average particle, 即后向散射比为 $1.9 \%$; NPSS 相位函数为 Kopelevich, 即后向散射比为 $3.7 \%$, 此两种相位函 数描述详见文献 $[6]$; 共 21 个波段, 分别为: $400-660 \mathrm{~nm}$ 每隔 $20 \mathrm{~nm}, 675 、 685 、 695 、 705 、 715 、 725 、 740 \mathrm{~nm}$.

利用 Hydrolight 模型和 Lee 模型分别模拟表 2 所示所有水体的反射率光谱数据 $r_{\mathrm{r} s} 、 R_{\mathrm{rs}}$, 以 Hydrolight 软 件模拟结果为真值, 对 Lee 深水生物光学模型模拟精度做出评价. 精度评价指标采用平均相对误差 (Average Relative Error, $A R E)$,可表示为:

$$
A R E=\left(\text { average }\left|\frac{x_{\mathrm{H}}-x_{\mathrm{L}}}{x_{\mathrm{H}}}\right|\right) \%
$$


表 2 四种不同组分的水体

Tab. 2 Four types of water with different components

\begin{tabular}{|c|c|c|c|}
\hline 类型 & 叶绿素 a 浓度 $\left(\mathrm{mg} / \mathrm{m}^{3}\right.$ ) & 可溶性有机物浓度 $\left(\mathrm{m}^{-1}\right)$ & 非藻类悬浮物浓度 $\left(\mathrm{g} / \mathrm{m}^{3}\right)$ \\
\hline I & $\begin{array}{l}0.5,1,5-100 \text { 每隔 } 5,100-220 \text { 每隔 } \\
10,220-300 \text { 每隔 } 20\end{array}$ & 0 & 0 \\
\hline II & 0 & $0.05,0.1-1$ 每隔 $0.1,1.5-9$ 每隔 0.5 & 0 \\
\hline III & 0 & 0 & $\begin{array}{l}0.5,1,5-100 \text { 每隔 } 5,100-220 \\
\text { 每隔 } 10,220-300 \text { 每隔 } 20\end{array}$ \\
\hline IV & $\begin{array}{l}0.5,1,5,10,20,30,40,60,80,100, \\
120,150,180,210,240,270,300\end{array}$ & $0.1,0.3,0.5,0.7,1,2,3,4,5,7,9$ & $\begin{array}{l}0.5,1,5,10,20,30,40,60,80, \\
100,120,150,180,210,240\end{array}$ \\
\hline
\end{tabular}

式中, $x_{\mathrm{H}}, x_{\mathrm{L}}$ 分别为 Hydrolight 模型与 Lee 模型模拟结果, average 表示每种水体下所有波段对应误差的平均.

\section{3 结果与讨论}

\section{1 Lee 模型模拟精度与水体组分浓度的关系}

I、II 、III种水体分别为除纯水外只含有浮游植物、CDOM、NPSS 的水体, 以下称为二组分水体 (表 2). 在此类水体下 Lee 模型模拟 $r_{\mathrm{rs}}$ 的平均相对误差 ARE 与叶绿素 a 浓度、CDOM 吸收系数、NPSS 浓度关系表 明, 当叶绿素 a 浓度为 $0.5-15 \mathrm{mg} / \mathrm{m}^{3}$ 时, Lee 模型模拟刚好处于水面以下遥感反射比 $r_{\mathrm{rs}}$ 的平均相对误差 $A R E$ 随着叶绿素 a 浓度增大先降低后增加, 其值为 $6.09 \%-5.08 \%$, 叶绿素 a 浓度为 $1 \mathrm{mg} / \mathrm{m}^{3}$ 时, $A R E$ 最小为 $2.02 \%$; 当叶绿素 a 浓度为 $15-300 \mathrm{mg} / \mathrm{m}^{3}$ 时, $A R E$ 随叶绿素浓度增加而降低, 其值为 $5.08 \%-3.71 \%$. 对于 $\mathrm{CDOM}$, 当其浓度小于 $0.5 \mathrm{~m}^{-1}$ 时, ARE 变化略有增加外, 其余浓度范围 $A R E$ 稳定在 $23.50 \%$ 左右. 而对于 NPSS, 其浓度变化与 $A R E$ 关系较为明显, $A R E$ 随着 NPSS 浓度增加而增加, 其值为 3.85\%-14.84\% (图 1a).
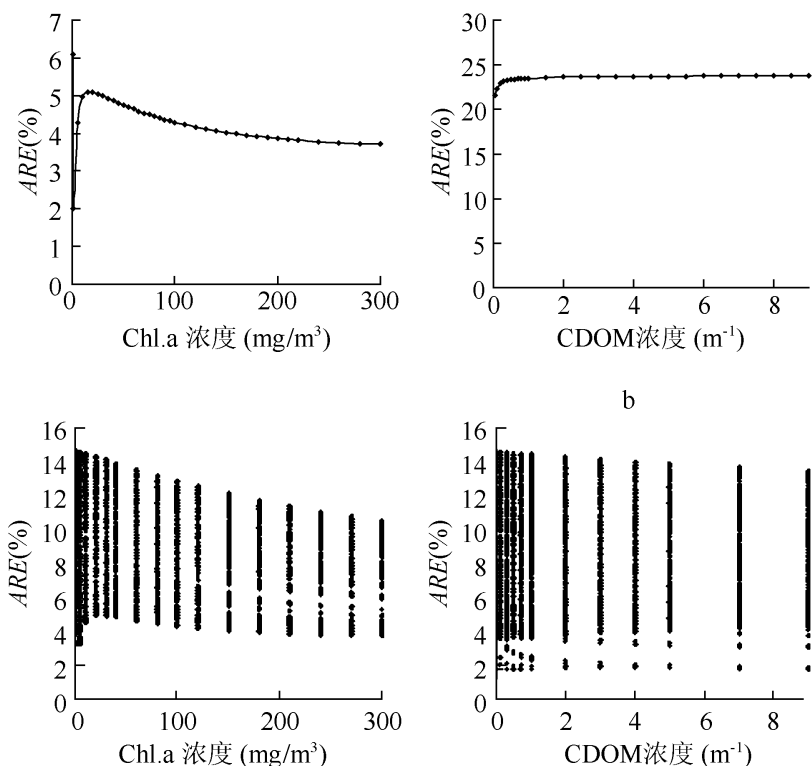

b
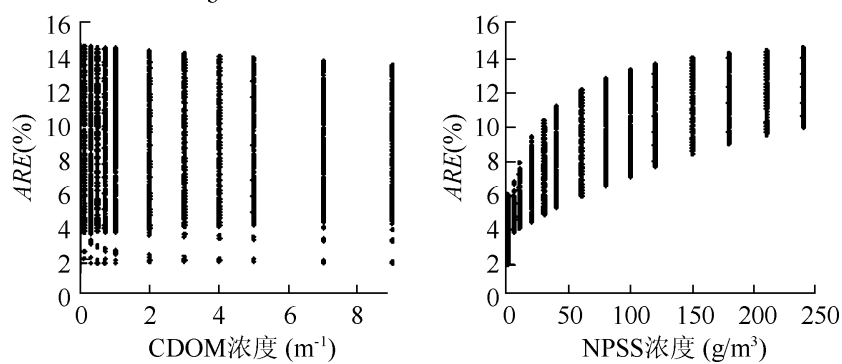

图 1 Lee 深水生物光学模型模拟二组分水体 (a) 和四组分水体 (b) $r_{\mathrm{rs}}$ 的平均相对误差 $A R E$ 与 叶绿素 a 浓度、可溶性有机物浓度、非藻类悬浮物浓度的关系

Fig. 1 Relationships of $A R E$ of $r_{\mathrm{rs}}$ in two-component waters( a ) and four-component waters(b) simulated by Lee deep-water bio-optical model versus: chlorophyll-a concentrations, CDOM concentrations, NPSS concentrations 
由以上结果可以看出: Lee 模型对于除纯水外仅含浮游植物的水体的 $r_{\mathrm{rs}}$ 模拟能力最强, 在 $0-300 \mathrm{mg} / \mathrm{m}^{3}$ 整个叶绿素 a 浓度范围内其模拟误差均在可接受范围内 (10\% 范围内); 对于除纯水外仅含 CDOM 的水体, 其模拟能力很差, 其模拟误差均不能接受; 而对于除纯水外仅含 NPSS 的水体, 其模拟能力随着 NPSS 浓度变 化而变化. 以上结果的主要原因是: Lee 等 $^{[6]}$ 在参数化其生物光学模型时, 其所选水体组分除纯水外仅浮游 植物、CDOM, 认为水体总吸收系数为纯水、浮游植物、CDOM 的吸收系数之和, 水体总后向散射系数为纯水 与浮游植物的后向散射系数之和, 并没有考虑 NPSS 的影响. 而对于 CDOM, 其后向散射系数往往认为为 0 , 所以其模拟精度较低.

以上二组分水体只是为了研究 Lee 模型对各种组分的模拟能力而设计的理想水体. 然而, 真实二类水体 的生物光学特性是纯水、浮游植物、CDOM、NPSS 四种组分相互作用的结果, 为此, 设计了如表 2 所示类型 IV 水体, 以下称为四组分水体, 共 17 种叶绿素 a 浓度、11 种 CDOM 浓度、15 种 NPSS 浓度, $17 \times 11 \times 15=2805$ 个样本.

对于四组分真实二类水体, 由于组分之间相互作用, Lee 模型模拟刚好处于水面以下遥感反射比 $r_{\mathrm{rs}}$ 的平 均相对误差 $A R E$ 与各组分浓度的关系仅为一种趋势, 由此不能判定其模型适用的组分浓度范围 (图 $1 \mathrm{~b}$ ). 然 而, 模拟四组分水体和二组分水体所示趋势一致, 即影响 Lee 模型模拟精度的只是叶绿素 a 浓度与 NPSS 浓 度, 而与 CDOM 几乎无关. 因此, 我们主要分析 Lee 深水生物光学模型模拟误差与叶绿素 a 浓度、NPSS 浓度 双变量的关系. 对实验设置的有限个样本的模拟误差 $(A R E)$ 在叶绿素 a 浓度、NPSS 浓度双变量空间进行插 值 ( $x$ 表示本次实验分析数据) (图 $2 \mathrm{a}$ ).

对于浮游植物主导型水体 Lee 模型具有很好的模拟精度, 而对于 NPSS 主导型水体其模拟误差较大, 最 高达到 14.62\% (图 2a). 以平均相对误差 $A R E \approx 10 \%$ (图 2a 中直线所示) 为基准, 可以大致界定 Lee 深水生 物光学模型的适用范围, 当水体中叶绿素 a 浓度与 NPSS 浓度满足: NPSS $<0.57 \mathrm{Chl} . \mathrm{a}+40$ 时, 其模型具有很 好的模拟精度; 当 NPSS $>0.57 \mathrm{Chl} . \mathrm{a}+40$ 时, 模型模拟精度较低.

然而, 对于一般的二类水体而言, 高 NPSS 浓度的高浑浊水体比较少见, 因而在大多数情况下 Lee 模型 是适用的.

\section{2 Lee 模型模拟精度的波谱特征}

以上分析的模拟误差均是各个波段模拟误差的平均值, 然而, 研究叶绿素 a 浓度反演算法常用波段的 模拟误差更具有实际意义. 为此, 在 Lee 模型模拟波段平均相对误差较小 $(A R E<10 \%)$ 的情况下, 本实验研 究了其模拟误差在波段上的分布 (图 $2 \mathrm{~b}$ ). 可以发现叶绿素 a 浓度反演算法常用波段, 如三波段模型、比值 法、峰高法、峰位法等叶绿素 a 浓度反演算法常用波段 $675 、 685 、 695 、 705 、 715 、 725 、 740 \mathrm{~nm}$ 处模拟误差相对其 他波段较大, 其中波段 $705 \mathrm{~nm}$ 模拟误差最大, 为 $7.57 \%$. 然而, 由于各种叶绿素 a 浓度反演模型固有的模型 反演误差, 使得对使用这些波段进行叶绿素 $\mathrm{a}$ 浓度反演后的反演误差难以给出一致、准确的估计, 故此处只 给出波段模拟误差.

\section{3 刚好在水面以上的遥感反射率的模拟精度}

水体反射率光谱实地采样时, 往往容易计算水面以上遥感反射比 $R_{\mathrm{rs}}$, 大多数经验/半经验叶绿素浓度反 演算法也是基于 $R_{\mathrm{rs}}$. 为此, 本研究对 $R_{\mathrm{rs}}$ 做了类似与 $r_{\mathrm{rs}}$ 的模拟误差分析, 其结果与 $r_{\mathrm{rs}}$ 模拟误差类似, 影响 $R_{\mathrm{rs}}$ 模拟精度的只是叶绿素 a 浓度与 NPSS 浓度, 而与 CDOM 几乎无关, 其模拟误差与叶绿素 a 浓度、NPSS 浓度 关系可以看出, $R_{\mathrm{rs}}$ 模拟误差随着水体中 NPSS 浓度的增大而增大. 然而相比 $r_{\mathrm{rs}}$ 模拟误差, $R_{\mathrm{rs}}$ 模拟误差减少很 多, 其最大模拟 $A R E$ 为 $8.16 \%$, 所有样本的 $A R E$ 为 $2.98 \%$, 可以认为 Lee 模型对 $R_{\mathrm{rs}}$ 具有很好的模拟精度 (图 3a). 同样对模拟误差在波段上的分布做了分析(图 3b), 结果显示在叶绿素 a 浓度反演算法常用波段其 模拟误差相对其他波段也比较大,最大 $A R E$ 波段为 $705 \mathrm{~nm}$,其误差值为 $4.60 \%$.

\section{4 结论}

本文通过设计大量不同组分生物光学特性的水体, 利用 Lee 模型和 Hydrolight 模型模拟刚好处于水面 以下遥感反射比 $r_{\mathrm{rs}}$ 和刚好处于水面以上遥感反射比 $R_{\mathrm{rs}}$, 以平均相对误差 $A R E$ 作为精度评价指标, 对 Lee 模 型模拟精度做出了相应的分析. 主要结论为: (1) 除非藻类悬浮物主导型水体外, Lee 模型对于一般二类水 

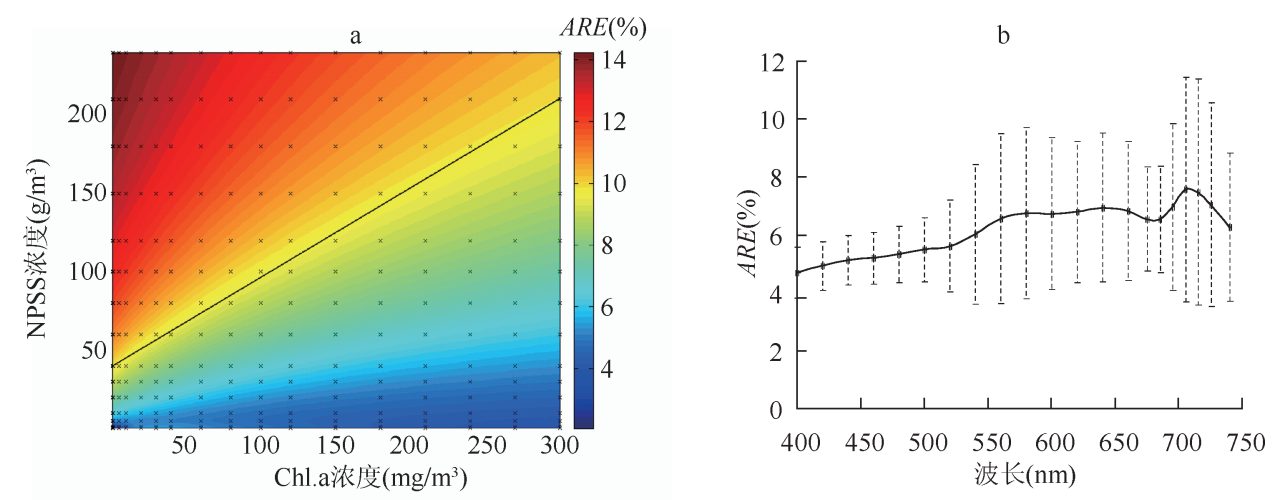

图 2 Lee 模型模拟 $r_{\mathrm{rs}}$ 的平均相对误差 $A R E$ 与叶绿素 a 浓度、非藻类悬浮物浓度的关系 (a)

以及 $A R E$ 在波段上的分布 $(\mathrm{b})$

Fig. 2 Relationships of $A R E$ of $r_{\mathrm{rs}}$ simulated by Lee deep-water bio-optical model versus chlorophyll-a concentrations and NPSS concentrations(a), distribution of ARE in each band(b)
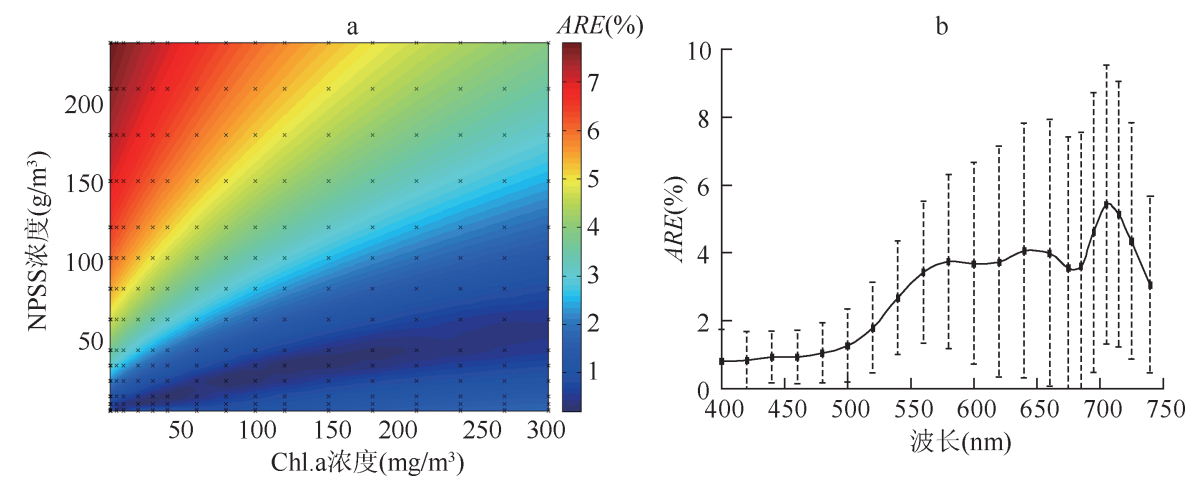

图 3 Lee 模型模拟 $R_{\mathrm{rs}}$ 平均相对误差 $A R E$ 与叶绿素 a 浓度、非藻类悬浮物 浓度的关系 $(\mathrm{a})$ 以及 $A R E$ 在波段上的分布 $(\mathrm{b})$

Fig. 3 Relationships of $A R E$ of $R_{\mathrm{rs}}$ simulated by Lee deep-water bio-optical model versus chlorophyll-a concentrations and NPSS concentrations(a), distribution of ARE in each band(b)

体具有很好的适用性. (2) 从模拟误差在波段上的分布可以看出叶绿素 a 浓度反演算法常用波段光谱模拟 误差较大. (3) $R_{\mathrm{rs}}$ 误差分析结果与 $r_{\mathrm{rs}}$ 类似. 本研究的重点在于模型的理论比较,在以后的工作中我们将采集 真实测量数据对模型精度进行进一步的检验.

\section{5 参考文献}

[ 1 ] Gitelson A. The Peak near 700nm on radiance spectra of algae and water-relationships of its magnitude and position with chlorophyll concentration. International Journal of Remote Sensing, 1992, 13: 3367-3373.

[ 2 ] Schalles JF, Gitelson A, Yacobi YZ et al. Estimation of chlorophyll a from time series measurements of high spectral resolution reflectance in an eutrophic lake. Journal of Phycology, 1998, 34 : 383-390.

[ 3 ] Gitelson A, Yacobi YZ, Schalles JF et al. Remote estimation of phytoplankton density in productive waters. Limnology and Lake Management, 2000, 55: 121-136.

[ 4 ] Gons HJ, Rijkeboer M, Bagheri S et al. Optical teledetection of chlorophyll a in estuarine and coastal waters. Environmental Science \& Technology, 2000, 34 : 5189-5192.

[ 5 ] Gordon HR, Brown OB, Jacobs MM. Computed relationships between the inherent and apparent optical properties of a flat 
homogeneous ocean. Appl Opt, 1975, 14(2) : 417-427.

[ 6 ] Lee Z, Carder KL, Mobley CD et al. Hyperspectral remote sensing for shallow waters: 1. A semianalytical model. Appl Opt, 1998, 37(27) : 6329-6338.

[ 7 ] Lee Z, Carder KL, Mobley CD et al. Hyperspectral remote sensing for shallow waters: 2. Deriving bottom depths and water properties by optimization. Appl Opt, 1999, 38(18) : 3831-3843.

[ 8 ] Lee Z, Carder KL. Absorption spectrum of phytoplankton pigments derived from hyperspectral remote-sensing reflectance. Remote Sensing of Environment, 2004, 89: 361-368.

[ 9 ] Cannizzaro JP, Carder KL. Estimating chlorophyll a concentrations from remote-sensing reflectance in optically shallow waters. Remote Sensing of Environment, 2006, 101: 13-24.

[10］杨 伟,陈 晋,松下文经. 基于生物光学模型水体叶绿素浓度反演算法. 光谱学与光谱分析,2009,29(1):38-42.

[11］马荣华,戴锦芳. 应用实测光谱估测太湖梅梁湾附近水体叶绿素浓度. 遥感学报,2005,9(1):78-85. 